\title{
Potential of interferon- $\gamma$-inducible protein 10 in improving tuberculosis diagnosis in HIV-infected patients
}

\section{To the Editors:}

In patients latently infected with Mycobacterium tuberculosis, immunosuppression significantly augments the risk of progression to active tuberculosis (TB) and TB is still one of the most frequent opportunistic infections worldwide. Prevention of TB in HIV-positive patients using the tuberculin skin testing (TST) followed by targeted preventive treatment is an effective strategy, but has thus far shown limited clinical success, in part due to the lack of a reliable test for latent TB infection (LTBI) [1]. Interferon (IFN) $\gamma$ release assays (IGRAs) have shown great potential in the improvement of LTBI diagnosis, but the tests perform suboptimally in immunocompromised populations. We and others have previously shown that HIV-positive patients have high rates of indeterminate QuantiFERON ${ }_{\mathbb{R}}-\mathrm{TB}$ Gold In-Tube test (QFT-IT) results and that this, at least in part, is due to low CD4 T-cell count [2-4].

We and others have also shown that IFN- $\gamma$-inducible protein 10 (IP-10/CXCL-10) is an alternative biomarker may improve immunodiagnosis of $M$. tuberculosis infection. IP-10 is induced specifically and in large quantities upon in vitro stimulation of whole blood with $M$. tuberculosis-specific antigens. We have developed an IP-10 based test [5] and shown that the IP-10 test performs comparably to the QFT-IT in adult patients with intact immune function [6].

We compared the sensitivity of an IP-10 based test with the QFT-IT for diagnosing $M$. tuberculosis infection in HIV-negative and HIV-positive patients with culture-confirmed active pulmonary tuberculosis (PTB). 300 patients newly diagnosed with PTB were recruited prospectively through the National Tuberculosis and Leprosy Programme (Mwanza, Tanzania). Blood was drawn for IGRA and HIV testing, and CD4 cell counts. Sputum was collected for microscopy and M. tuberculosis culture. Patients $>15$ yrs of age with a positive sputum culture result were enrolled. All HIV-positive patients were newly diagnosed in the study context. A detailed description of recruitment, testing and sample handling has been published previously [3]. After QFT-IT testing, supernatants were frozen at $-80^{\circ} \mathrm{C}$. $1 \mathrm{yr}$ later, samples were thawed and analysed for IP-10 using xMAP technology on the Luminex platform (Luminex Corp., Austin, TX, USA) as described previously [5]. Algorithms for interpretation of IP-10 test results have been developed previously using receiver operating characteristic analysis [5]; however, the mitogen cut-off was chosen arbitrarily (see online supplement 1). One IU corresponds to $50 \mathrm{pg}$ IFN- $\gamma$. In the literature, there is a lack of consensus on how to present IGRA sensitivity and specificity: including or excluding indeterminate results. We suggest the designations "sensitivity" for calculation after exclusion of indeterminate results, and "positivity rate" for calculation amongst all possible test outcomes. High rates of indeterminate results (i.e. low positivity rate) mainly affect usability and cost-effectiveness, and may impair the feasibility of implementing the test in severely immunocompromised individuals. Positivity rate was calculated among all possible tests outcomes (i.e. including indeterminate results), whereas sensitivity was calculated after exclusion of indeterminate test results. All comparisons were done using nonparametric paired tests. A $p$-value $\leqslant 0.05$ was considered significant. Permission to conduct the study was granted by the ethics committee of the National Institute for Medical Research (Mwanza, Tanzania) and was approved by The Danish National Committee on Biomedical Research Ethics (Copenhagen, Denmark; reference 2005-7041-57).

HIV-negative $(n=92)$ and HIV-positive $(n=65)$ patients were included in the study. HIV-positive patients were older, had lower CD4 cell counts and were more likely to be female ( $p<0.05$ for all, see online supplement 2$)$. The IP-10 test had comparable positivity rate and sensitivity to the QFT-IT in both HIV-positive and HIV-negative patients. Positivity rate was lower and indeterminate rate higher in HIV-positive patients for both tests, while there was no significant difference in sensitivity for either test (table 1).

Impaired performance of the QFT-IT in HIV-positive patients with a low CD4 cell count has been well documented and we have previously demonstrated that QFT-IT sensitivity decreases with decreasing CD4 cell count in HIV-positive patients, which is mediated by an increasing proportion of indeterminate results. Interestingly, for the IP-10 test, we found no such trend with CD4 cell count for rate of positive $(p=0.44)$ or for indeterminate $(p=0.55)$ results (fig. 1$)$. Another recent study in HIV-infected patients with active TB reported that, while QFT-IT yielded indeterminate results when CD4 cell count was $<200$ cells $\cdot \mu \mathrm{L}^{-1}$, an IP-10-based test yielded indeterminate results only when CD4 cell count was $<50$ cells $\mu \mathrm{L}^{-1}$ [4]. Thus, it seems that IP-10 is not, or at least is much less, affected by CD4 cell count than is the QFT-IT. Although IFN- $\gamma$ is directly involved in inducing IP-10 production, IP-10 is produced primarily by the monocyte and might be induced by CD4 T-cell- and IFN- $\gamma$-independent pathways (e.g. induction 


\begin{tabular}{|c|c|c|c|}
\hline TABLE 1 & $\begin{array}{l}\text { Distribution of Quan } \\
\text { (QFT-IT) and interfer } \\
\text { (IP)-10 test results a } \\
\text { tests }\end{array}$ & $\begin{array}{l}\text { IFERON } N_{\mathbb{R}} \text {-TB } \\
\text { on- } \gamma \text {-inducible } \\
\text { hd comparisor }\end{array}$ & $\begin{array}{l}\text { Gold In-Tube } \\
\text { protein } \\
\text { between }\end{array}$ \\
\hline \multirow[t]{2}{*}{ Test result } & \multicolumn{2}{|c|}{ Single-marker tests } & \multirow[t]{2}{*}{ Combined test } \\
\hline & IP-10 test ${ }^{\#}$ & QFT-IT" & \\
\hline \multicolumn{4}{|l|}{ HIV-negative $^{+}$} \\
\hline Positive & 75 (82; 72-89) & $74(80 ; 71-88)$ & $86(93 ; 88-99)^{\# \#}$ \\
\hline Sensitivity & $86(79-93)$ & $88(81-95)$ & $96(91-99)^{\# \#}$ \\
\hline Negative & $12(13 ; 7-22)$ & $10(11 ; 5-19)$ & $4(4 ; 0-9)^{\# \#}$ \\
\hline Indeterminate & $5(5 ; 2-12)$ & $8(9 ; 4-16)$ & $2(2 ; 0-5)^{\pi}$ \\
\hline \multicolumn{4}{|l|}{ HIV-positive $e^{\S}$} \\
\hline Positive & $41(63 ; 50-75)^{f}$ & $41(63 ; 50-75)^{f}$ & $46(71 ; 60-82)^{\# \#}$ \\
\hline Sensitivity & $76(65-87)$ & $82(74-95)$ & $88(80-97)^{\# \#}$ \\
\hline Negative & $13(20 ; 11-32)$ & $9(14 ; 7-25)$ & $6(9 ; 2-16)^{++}$ \\
\hline Indeterminate & $11(17 ; 9-28)^{f}$ & $15(23 ; 14-35)^{f}$ & $13(20 ; 10-30)$ \\
\hline
\end{tabular}

Data are presented as $\mathrm{n}(\% ; 95 \% \mathrm{Cl})$ or $\%(95 \% \mathrm{Cl})$. Sensitivity was calculated after excluding indeterminate results. Algorithms for test results can be seen in online supplement 1. In the combined approach, the test was deemed positive if either test was positive, negative if both tests were negative and indeterminate if one test was indeterminate and the other was negative. All p-values were calculated using McNemar's test. ${ }^{*}$ : cut-off $673 \mathrm{pg} \cdot \mathrm{mL}^{-1}$, as determined in [5]; ?: cut-off $0.35 \mathrm{IU} \cdot \mathrm{mL}^{-1}\left(17.5 \mathrm{pg} \cdot \mathrm{mL}^{-1}\right) ;{ }^{+}: \mathrm{n}=92{ }^{5}: \mathrm{n}=65 ;{ }^{f}: \mathrm{p}<0.05$ compared with HIV-negative patients; ${ }^{\# \#: ~} \mathrm{p}<0.05$ compared with either single-marker test; " : $p<0.05$ compared with the QFT-IT alone; ${ }^{++}:$p $<0.05$ compared with the IP-10 test alone.

by other cytokines) [7]. We found no effect of monocyte count on IP-10 test performance in either HIV-positive or -negative patients (data not shown). Another possible explanation for the finding is the larger amounts of biomarker produced, which may render an IP-10-based test less sensitive to the effect of immune suppression. Antigen-induced IP-10 levels were 21fold higher than IFN- $\gamma$ (interquartile range 7-48; $\mathrm{p}<0.001$ ). Lastly, the mitogen cut-off was rather low, giving less indeterminate IP-10 test results and, thereby, possibly obscuring CD4 dependency. However, increasing the mitogen cut-off did not change these findings (data not shown). We found no further connection between clinical characteristics (listed in online supplement 2) and indeterminate or false negative results by either test.

Agreement between the tests was $0.73(\kappa=0.67)$ for HIVnegative and $0.78(\kappa=0.60)$ for HIV-positive patients (online supplement 3). Combining the tests significantly increased sensitivity to $96 \%$ in HIV-negative and $88 \%$ in HIV-positive patients (table 1). We and others have found a similar improvement in sensitivity among patients from low-endemic setting without a compromise in specificity $[8,9]$. Thus, combining tests appears to be a feasible way to improve performance of the antigen-specific assays. We found no connection between clinical characteristics (listed in online supplement 2) and discordant test results.

It is important to remember that the diagnostic sensitivity obtained in a population with active and severe TB may be lower than the sensitivity obtained among otherwise healthy individuals with LTBI. Also, a limitation of the present study is the lack of a control group, which prevents estimation of specificity. None of the tests discriminates between active TB and LTBI and, given the prevalence of LTBI in the background population, we could not identify a valid control group in this setting. Inclusion of a control group from another geographic region would not have been a valid approach due to potential influences of genetic background, comorbidity, nutritional status, etc. However, a very high specificity of both QFT-IT and the IP-10 test has been convincingly demonstrated in healthy, unexposed individuals from low-endemic regions. Unfortunately, TST was not performed in this study population.
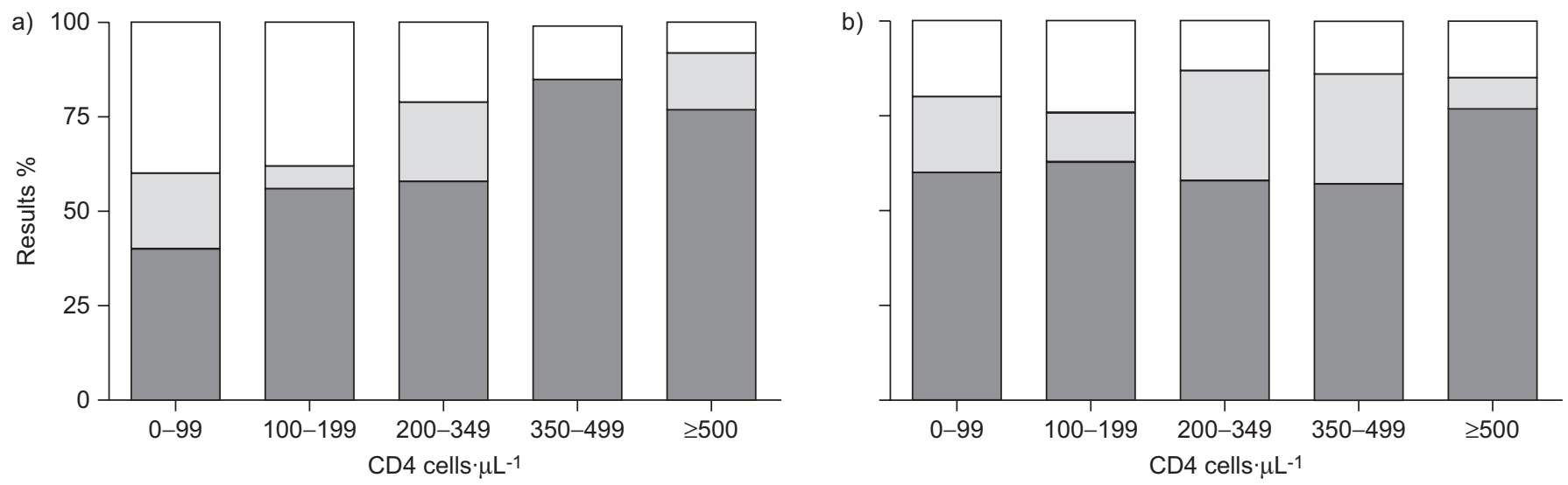

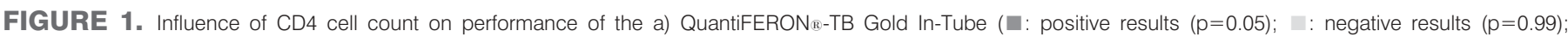
$\square$ : indeterminate results $(p=0.04)$ ) and $b)$ interferon- $\gamma$-inducible protein 10 tests ( $\square$ : positive results $(p=0.44)$; $\square$ : negative results $(p=0.72)$; $\square$ : indeterminate results $(p=0.55))$ in HIV-positive patients. $p$-values are for Cochrane-Armitage test for trend. Test results were grouped by the individual number of $C D 4$ cells $\mu \mathrm{L}^{-1}$. The numbers of patients in each CD4 cell group were as follows. 0-99 cells $\mu \mathrm{L}^{-1}: n=5 ; 100-199$ cells $\cdot \mu \mathrm{L}^{-1}: n=16 ; 200-349$ cells $\mu \mu \mathrm{L}^{-1}: n=24 ; 350-499 \mathrm{cells} \cdot \mu \mathrm{L}^{-1}: \mathrm{n}=7 ;>500 \mathrm{cells} \cdot \mu \mathrm{L}^{-1}$ : $\mathrm{n}=13$. a) Modified from [3] 
In conclusion, the IP-10 test performs with equal sensitivity to the QFT-IT in a TB/HIV-endemic setting. Combining the tests significantly improves sensitivity, even in HIV-positive patients. The IP-10 test seems less affected by a low CD4 cell count than the QFT-IT. Further approaches for improvement of TB diagnosis are needed, especially in TB/HIV-endemic settings, and the IP-10 biomarker represents a promising example.

M.G. Aabye ${ }^{*, * *}$, M. Ruhwald****, G. PrayGod ${ }^{\#}$, K. Jeremiah ${ }^{\# \text {, }}$ M. Faurholt-Jepsen ", D. Faurholt-Jepsen ", N. Range ${ }^{+}$, H. Friis ${ }^{\Uparrow}$, J. Changalucha", A.B. Andersen ${ }^{\S}$ and P. Ravn ${ }^{f}$ ${ }^{*}$ Clinical Research Center, University of Copenhagen, Hvidovre Hospital, Hvidovre, "Dept of Human Nutrition, Faculty of Life Sciences, University of Copenhagen, Frederiksberg, ${ }^{5}$ Dept of Infectious Diseases, University of Copenhagen, Rigshospitalet, Copenhagen, and ${ }^{f}$ Unit for Infectious Diseases, University of Copenhagen, Herlev Hospital, Herlev, Denmark. "National Institute for Medical Research, Mwanza Medical Research Centre, Mwanza, and ${ }^{+}$National Institute for Medical Research, Muhimbili Medical Research Centre, Dar Es Salaam, Tanzania. **Both authors contributed equally to the study.

Correspondence: M.G. Aabye, Clinical Research Centre, University of Copenhagen, Hvidovre Hospital, Kettegaard Alle 30, 2650 Hvidovre, Denmark. E-mail: martine@aabye.com

Support Statement: Data from this study were presented, in part, at the 2nd Global Symposium on IGRAs, (Dubrovnik, Croatia; 2009), the European Respiratory Society 19th Annual Congress, (Vienna, Austria; 2009) and the 17th Conference on Retroviruses and Opportunistic Infections (CROI) (New Orleans, LA, USA; 2010).

Statement of Interest: Statements of interest for M.G. Aabye, M. Ruhwald and P. Ravn, and the study itself can be found at www.erj.ersjournals.com/site/misc/statements.xhtml
Acknowledgements: The authors would like to thank all colleagues contributing to this project, including the staff at the National Institute for Medical Research (NIMR) Mwanza Centre, Sekou Toure Regional Hospital, Buzuruga Health Centre, Bugando Medical Centre and Butimba Health Centre (all Mwanza, Tanzania), especially laboratory technician O. Kaswamila (NIMR Mwanza Centre). Equally, the authors thank the staff at the Laboratory for Infectious Diseases at University of Copenhagen, Hvidovre Hospital (Hvidovre, Denmark) and at the AIDS Laboratory at University of Copenhagen, Rigshospitalet (Copenhagen, Denmark) for their collaboration, as well as R. Wallis (Pfizer, New London, CT, USA) for his helpful comments and critique.

\section{REFERENCES}

1 World Health Organisation. Global tuberculosis control: epidemiology, strategy, financing. Geneva, WHO, 2009.

2 Domínguez J, Latorre I, Altet $\mathrm{N}$, et al. IFN- $\gamma$-release assays to diagnose TB infection in the immunocompromised individual. Expert Rev Resp Med 2009; 3: 309-327.

3 Aabye MG, Ravn P, PrayGod G, et al. The impact of HIV infection and CD4 cell count on the performance of an interferon gamma release assay in patients with pulmonary tuberculosis. PLOS ONE 2009; 4: e4220.

4 Kabeer BS, Sikhamani R, Raja A. Comparison of interferon gamma and interferon gamma-inducible protein-10 secretion in HIVtuberculosis patients. AIDS 2010; 24: 323-325.

5 Ruhwald M, Bodmer T, Maier C, et al. Evaluating the potential of IP10 and MCP-2 as biomarkers for the diagnosis of tuberculosis. Eur Respir J 2008; 32: 1607-1615.

6 Ruhwald M, Petersen J, Kofoed K, et al. Improving T-cell assays for the diagnosis of latent TB infection: potential of a diagnostic test based on IP-10. PLOS ONE 2008; 3: e2858.

7 Korpi-Steiner NL, Bates ME, Lee WM, et al. Human rhinovirus induces robust IP-10 release by monocytic cells, which is independent of viral replication but linked to type I interferon receptor ligation and STAT1 activation. J Leukoc Biol 2006; 80: 1364-1374.

8 Lighter J, Rigaud M, Huie M, et al. Chemokine IP-10: an adjunct marker for latent tuberculosis infection in children. Int J Tuberc Lung Dis 2009; 13: 731-736.

9 Syed Ahamed KB, Raman B, Thomas A, et al. Role of QuantiFERONTB gold, interferon gamma inducible protein-10 and tuberculin skin test in active tuberculosis diagnosis. PLoS ONE 2010; 5: e9051.

DOI: $10.1183 / 09031936.00039010$

\section{New insight into extremely drug-resistant tuberculosis: using atomic force microscopy}

\section{To the Editors:}

We have recently documented the emergence of new forms of resistant tuberculosis (TB) bacilli (totally drug resistant (TDR)TB or extremely drug-resistant (XXDR)-TB strains) among multidrug-resistant (MDR)-TB patients [1]. XXDR-TB defines any case of TB with resistance to all first- and second-line antiTB drugs whose smears and cultures remain positive despite prolonged therapy [1-3]. At the cellular level of XXDR-TB strains, adaptation was observed and evaluated using transmission electron microscopy (TEM) $[4,5]$. In the exponential phase, three different cell populations were clearly distinguished: one displayed an ordinary pattern $(70-80 \%)$, one exhibited a round or oval shape (15-20\%), and the third displayed an extraordinarily thick cell wall $(21-26 \mathrm{~nm})$ with features similar to stationary or anaerobic dormant bacilli (5$7 \%)[3,4]$. These adapted forms were detected in all XXDR-TB isolates, irrespective of their super families or genotype patterns. We tried to evaluate the different cell population of XXDR-TB strains in comparison to susceptible cells using atomic force microcsopy (AFM). To achieve this goal, we included sputum and culture positive specimens of the same 